\title{
Factors that Affect the Performance of COVID-19 Infection Management Administration in General Hospitals
}

\author{
Jinkyung Kim ${ }^{1}$, Mi Hyang Lee ${ }^{2}$, Woo Sok Han ${ }^{1}$, Jung Hee Park ${ }^{3}$ \\ ${ }^{1}$ Associate Professor, Department of Hospital Management, Konyang University, Daejeon, Korea, ${ }^{2}$ Associate \\ Professor, Department of Nursing, Konyang University, Daejeon, Korea, ${ }^{3}$ Assistant Professor, Department of \\ Emergency Medical Service, Konyang University, Daejeon, Korea; jhpug@konyang.ac.kr (J.H.P)
}

\begin{abstract}
Background: This study aimed to provide basic data for the development of COVID-19 infection management educational programs by confirming the factors that affect the performance of COVID-19 infection management administration in general hospitals.

Method: Data was collected from administrative staff at 192 general hospitals by means of a structuralized questionnaire, and SPSS Ver. 21.0 software was used for data analysis.

Conclusion: The extent of knowledge and execution of COVID-19 infection management processes by administrative staff in general hospitals was positively correlated with the extent of educational demand for COVID-19 infection management, with explanatory power of $16.7 \%$. It is necessary to include factors including COVID-19 transmission route, transmission prevention, and staff safety in COVID-19 infection management educational program administration. In addition, it is essential also to performance COVID-19 infection management education.
\end{abstract}

Keywords: COVID-19, Knowledge, Importance level, Educational demand, Performance.

\section{Introduction}

The outbreak of COVID-19 $9^{(1)}$ that began in Wuhan, China in December 2019 resulted in the first confirmed case in Korea on January 20, 2020. From that point on, it proliferated explosively in venues of communal life including religious organizations, clubs, mental hospitals, nursing hospitals and nursing homes, and call-centers ${ }^{(2)}$. In particular, since patients with underlying diseases and degraded immunity tend to be hospitalized at medical institutions, mass infection at medical institutions imparted enormous effects not only on patients and guardians but also medical staff, administrative staff,

\section{Corresponding Author:}

\section{Mi Hyang Lee}

Department of Nursing, College of Nursing, Konyang University, 158, Gwanjeodong-ro, Seo-gu, Daejeon, South Korea

e-mail: haha@konyang.ac.kr and medical technicians. At the time of the outbreak of Middle East Respiratory Syndrome (MERS) in Korea in 2015, the proportion of medical health staff infected among total cases was $18 \%$, with 30 confirmed cases from a total of $186^{(3)}$.

Since the outbreak of MERS in 2015, there have been studies conducted on nurses regarding the actual status and awareness of MERS infection management $t^{(4)}$, assessments of the knowledge, intention for infection management activities, and extent of educational demand for respiratory diseases among nurses (5) and studies of infection management prevention knowledge and extent of performance of said management prevention among nursing aids, care workers, and caregivers ${ }^{(6)}$. The majority of these studies have focused on nursing personnel, who generally had the longest period of close contact with patients. In addition, level D practical education on how to put on and take off quarantine gowns properly has been conducted for medical staff working at medical institutions throughout the country since the outbreak of MERS. However, the majority of 
such education is aimed at medical staff, with inadequate education provided for administrative staff.

In order to prevent infection at medical institutions, in is essential for all constituent members including physicians, nurses, medical technicians, and administrative staff to comply strictly with the infection prevention guidelines, including directions regarding hand hygiene, personal protective devices, and environmental management ${ }^{(7)}$. Workers at medical institutions represent the key channel for outbreaks of medically related infections. Since the extent of infection management performance will be elevated with higher awareness of infection management among such workers, infection management education is very important ${ }^{(8,9)}$. The administrative staff at medical institutions constitute personnel who provide the necessary support for patient treatment and care, including materials and system resources, and are the first to come in contact with patients during visits to medical institutions. All patients need to register with the accounts department first before receiving treatment and care. Since it is difficult to assess the state of patient respiratory infections at this time, administrative staff are confronted with very high possibility of being exposed to infection. Nonetheless, there has not been any study that investigated the actual state of the respiratory infection management knowledge among administrative staff until now.

Accordingly, this study aims to provide basic data for the development of educational programs customized for administrative staff by assessing COVID-19 infection management knowledge, importance level, extent of educational demand, and extent of performance among administrative staff working in medical institutions. The specific goals of the study are as follows. First, describe the general characteristics, COVID-19-related knowledge, importance level, extent of educational demand, and extent of execution among study subjects. Second, analyze differences in the execution of infection management in accordance with the general characteristics of the study subjects. Third, assess correlations between COVID-19 knowledge, importance level, and the extent of educational demand and the performance thereof. Fourth, analyze factors that affect the performance of COVID-19 infection management.

\section{Materials and Method}

Materials: This is a descriptive study aimed at assessing COVID-19 infection management knowledge, importance level, extent of educational demand, and extent of performance among administrative staff members working at 4 general hospitals in the D Region. Study data was collected over a period of approximately 3 weeks from November 4 to 25, 2020. After extracting data from 4 general hospitals in the D Region, the researcher personally visited these institutions to carry out a survey after first explaining the purposes and methods of the study and acquiring the consent of the administrative staff. Minimum numbers of subjects necessary for statistical analysis were calculated using G*power 3.1 Program. Computed with a significance level of $\alpha=0.05$, scale effect of $d=0.15$, test power of 0.95 , and 12 prediction variables, the minimum number of subjects was 184. A total of 200 questionnaires were distributed in consideration of the response rate and drop-out rate. Data collated from a total of 192 questionnaires retrieved after excluding 8 questionnaires filled in inappropriately were used for analysis.

A measurement tool was developed by the researcher in accordance with the study goals by making reference to the guidelines to cope with COVID-19 issued by the Korea Centers for Disease Control and Prevention. The questionnaire consisted of a total of 73 questions, comprising 11 questions on general characteristics, 20 questions on knowledge of COVID-19, 20 questions on the importance level of COVID-19, 8 questions on the extent of educational demand for COVID-19 infection management, and 14 questions on the execution of COVID-19 infection management. The final questionnaire was prepared after its content was verified by the administrative managers at the 4 general hospitals, 2 nursing college professors, and 3 nurses specializing in infection management. The Content Validity Index (CVI) exceeded 0.80 .

1. COVID-19 Knowledge: The survey tool for assessment of COVID-19 knowledge was composed of a total of 20 questions, with each question to be answered with either 'Yes', 'No', or 'Don't know'. 1 point was allocated for each correct answer, while 0 points were allocated for wrong answers and 'Don't know' responses. As such, the total score for each subject ranged from 0 to 20 points, with higher scores signifying that subjects had higher knowledge of COVID-19.

2. Importance Level of COVID-19: This section was composed of 20 questions with a 5-point Likert scale applied to each question. Higher scores 
signified higher importance placed by the subject on COVID-19. The reliability of the questionnaire as a tool of assessment was secured with a Cronbach alpha value of 0.94 in this study.

3. Extent of Educational Demand for COVID-19 Infection Management: This section was composed of 8 questions with a 4-point Likert scale applied to each question and a range of scores from 1 point for 'Not important at all' to 4 points for 'Very important'. Higher scores signified a higher extent of educational demand for COVID-19 infection management. The reliability of the questionnaire as a tool of measurement of the extent of educational demand for COVID-19 management was indicated by a Cronbach alpha value of 0.83 .

\section{Performance of COVID-19 Infection} Management: It is composed of 14 questions with 4-point Likert scale applied to each question with the range of scores of 1 point for 'Not performance at all' to 4 points for 'Always performance'. Higher score signifies higher level of execution of COVID-19 infection management. The reliability of the questionnaire as the tool for the measurement of the execution of COVID-19 infection management was secured with Cronbach's alpha value of 0.84 .

\section{Method}

The data collected was analyzed by means of the SPSS Ver. 21.0 program, employing the following analysis methods. Characteristics of study variables were analyzed by means of descriptive statistics, while differences in the study variables in accordance with general characteristics were analyzed using the t-test and ANOVA. Follow-up analysis was verified with the Duncan test, while the correlations among the study variables were analyzed with Pearson's Correlation Coefficient. In addition, Multiple Regression Analysis was used to analyze the factors affecting execution of the infection management by study subjects.

\section{Results and Discussion}

General Characteristics of Study subjects: The general characteristics of the study subjects are given in Table 1. There were 112 males (80\%) and 80 females $(41.7 \%)$ with an average age of $36.86 \pm 9.56$ years. Regarding their employment positions, team members were most numerous at $157(81.8 \%)$, while the average period of work experience was $10.78 \pm 8.76$ years. There were 110 subjects working in departments that come in direct contact with patients $(57.3 \%)$ and 128 subjects underwent respiratory infection management education during the last 1 year $(66.7 \%)$. 54 subjects came in contact with patients suspected of COVID-19 (28.1\%), while 6 subjects came into contact with confirmed COVID-19 cases (3.1\%).

Table 1. General Characteristics of Study Subjects $(n=192)$

\begin{tabular}{|c|c|c|c|c|}
\hline Categories & Variables & $\mathbf{N}$ & $\%$ & Mean \pm SD \\
\hline \multirow{2}{*}{ Gender } & Male & 112 & 80 & \\
\hline & Female & 80 & 41.7 & \\
\hline \multirow{3}{*}{ Age } & 20 's & 57 & 29.7 & \multirow{3}{*}{$36.86 \pm 9.56$} \\
\hline & 30 's & 62 & 32.3 & \\
\hline & Over 40 years & 73 & 38.0 & \\
\hline \multirow{3}{*}{ Marital status } & Single & 83 & 43.2 & \\
\hline & Married & 107 & 55.7 & \\
\hline & Others & 2 & 1.1 & \\
\hline \multirow{3}{*}{ Position } & Team member & 157 & 81.8 & \\
\hline & Part manager & 17 & 8.9 & \\
\hline & Higher than team manager & 18 & 9.4 & \\
\hline \multirow{2}{*}{ Work experience } & Less than 5 years & 63 & 32.8 & \multirow{2}{*}{$10.78 \pm 8.76$} \\
\hline & More than 5 years & 129 & 67.2 & \\
\hline
\end{tabular}




\begin{tabular}{|c|c|c|c|c|}
\hline Categories & Variables & $\mathbf{N}$ & $\%$ & $\operatorname{Mean} \pm$ SD \\
\hline \multirow{2}{*}{ Department } & Department with contact with patients & 110 & 57.3 & \\
\hline & Department with no contact with patients & 82 & 42.7 & \\
\hline \multirow{2}{*}{$\begin{array}{l}\text { Respiratory system infection management } \\
\text { education within the last } 1 \text { year }\end{array}$} & Yes & 128 & 66.7 & \\
\hline & No & 58 & 33.3 & \\
\hline \multirow{2}{*}{$\begin{array}{l}\text { Contact with patient suspected of } \\
\text { COVID-19 }\end{array}$} & Yes & 54 & 28.1 & \\
\hline & No & 138 & 71.9 & \\
\hline Contact with confirmed COVID-19 patient & Yes & 6 & 3.1 & \\
\hline
\end{tabular}

COVID-19 Knowledge, Importance Level, Extent of Educational Demand, and Infection Management Performance: The levels of COVID-19 knowledge, importance, extent of educational demand, and infection management performance by general hospital administrative staff are illustrated in Table 2 . The average score for COVID-19 knowledge among administrative staff was $14.54 \pm 2.69$ out of a possible 20 points, while those of the subordinate domains including epidemiology and infection management were $7.72 \pm 1.37$ and $6.88 \pm 1.94$ points, respectively. The average score for importance level was $4.09 \pm 0.88$ out of a possible 5 points, while those of the subordinate domains including epidemiology and infection management were $4.10 \pm 0.85$ and $4.09 \pm 0.98$ points, respectively. The average score for the extent of educational demand was $3.66 \pm 0.34$ out of a possible 4 points, while those of the subordinate domains including characteristics of disease, diagnosis, and examination, treatment methods, transmission route, transmission prevention, putting on and taking off of personal protective devices, isolation guidelines, lifting of patient isolation, and staff safety were $3.64 \pm 0.79, \quad 3.60 \pm 0.53, \quad 3.53 \pm 0.59, \quad 3.84 \pm 0.36$, $3.71 \pm 0.49,3.70 \pm 0.50,3.47 \pm 0.62$ and $3.78 \pm 0.43$ points, respectively. The average score for the performance of infection management was $3.40 \pm 0.58$ out of a possible 4 points, while those of the subordinate domains including hand hygiene, personal protective devices, environmental disinfection, respiratory etiquette, and infection management by staff were $3.22 \pm 0.34$, $3.55 \pm 0.86,2.73 \pm 1.21,3.63 \pm 0.64$ and $3.70 \pm 0.59$ points, respectively.

Table 2. COVID-19 Knowledge, Importance Level, Extent of Educational Demand and Infection Management Performance

\begin{tabular}{|c|c|c|c|}
\hline Categories & Subordinate domains & $\operatorname{Mean} \pm$ SD & Range \\
\hline \multirow{3}{*}{ Knowledge } & Epidemiology & $7.72 \pm 1.37$ & \multirow{2}{*}{$0 \sim 10$} \\
\hline & Infection management & $6.88 \pm 1.94$ & \\
\hline & Total & $14.54 \pm 2.69$ & $0 \sim 20$ \\
\hline \multirow{3}{*}{ Importance level } & Epidemiology & $4.10 \pm .85$ & \multirow{3}{*}{$1 \sim 5$} \\
\hline & Infection management & $4.09 \pm .98$ & \\
\hline & Total & $4.09 \pm .88$ & \\
\hline \multirow{9}{*}{$\begin{array}{l}\text { Extent of educational } \\
\text { demand }\end{array}$} & Characteristics of disease & $3.64 \pm .79$ & \multirow{9}{*}{$1 \sim 4$} \\
\hline & Diagnosis and examination & $3.60 \pm .53$ & \\
\hline & Treatment method & $3.53 \pm .59$ & \\
\hline & Transmission route and transmission prevention & $3.84 \pm .36$ & \\
\hline & Putting on and taking off of personal protective devices & $3.71 \pm .49$ & \\
\hline & Isolation guidelines & $3.70 \pm .50$ & \\
\hline & Lifting of patient isolation & $3.47 \pm .62$ & \\
\hline & Staff safety & $3.78 \pm .43$ & \\
\hline & Total & $3.66 \pm .34$ & \\
\hline
\end{tabular}




\begin{tabular}{|c|c|c|c|}
\hline Categories & Subordinate domains & $\operatorname{Mean} \pm$ SD & Range \\
\hline \multirow{6}{*}{ Performance } & Hand hygiene & $3.22 \pm .34$ & \multirow{6}{*}{$1 \sim 4$} \\
\hline & Personal protective devices & $3.55 \pm .86$ & \\
\hline & Environmental disinfection & $2.73 \pm 1.21$ & \\
\hline & Respiratory etiquette & $3.63 \pm .64$ & \\
\hline & Infection management by staff & $3.70 \pm .59$ & \\
\hline & Total & $3.40 \pm .58$ & \\
\hline
\end{tabular}

Differences in Study Variables in Accordance with General Characteristics: There were statistically significant differences in the performance of infection management in accordance with general characteristics depending on the department in which the subjects worked ( $\mathrm{t}=2.076, p=.039)$, with those working in departments with contact with patients displaying higher levels of performance of infection management in comparison to those working in departments with no contact with patients (Table 3 ).

Table 3. Extent of the Execution of COVID-19 Infection Management in Accordance with General Characteristics

\begin{tabular}{|c|c|c|c|}
\hline \multirow[t]{2}{*}{ Categories } & \multirow[t]{2}{*}{ Variables } & \multicolumn{2}{|c|}{$\begin{array}{c}\text { Extent of infection management } \\
\text { execution }\end{array}$} \\
\hline & & $\operatorname{Mean} \pm$ SD & t or F (p) \\
\hline \multirow{2}{*}{ Gender } & Male & $3.39 \pm .47$ & \multirow{2}{*}{$\begin{array}{l}-.467 \\
(.641)\end{array}$} \\
\hline & Female & $3.43 \pm .71$ & \\
\hline \multirow{3}{*}{ Age } & 20 's & $3.41 \pm .76$ & \multirow{3}{*}{$\begin{array}{c}.058 \\
(.944)\end{array}$} \\
\hline & 30 's & $3.41 \pm .44$ & \\
\hline & Over 40 years & $3.38 \pm .53$ & \\
\hline \multirow{3}{*}{ Marital status } & Single & $3.42 \pm .68$ & \multirow{3}{*}{$\begin{array}{l}.128 \\
(.880)\end{array}$} \\
\hline & Married & $3.38 \pm .50$ & \\
\hline & Others & $3.50 \pm .71$ & \\
\hline \multirow{3}{*}{ Position } & Team member & $3.39 \pm .35$ & \multirow{3}{*}{$\begin{array}{l}1.106 \\
(.333)\end{array}$} \\
\hline & Part manager & $3.67 \pm .34$ & \\
\hline & Higher than team manager & $3.74 \pm .26$ & \\
\hline \multirow{2}{*}{ Work experience } & Less than 5 yeas & $3.42 \pm .75$ & \multirow{2}{*}{$\begin{array}{c}.284 \\
(.777)\end{array}$} \\
\hline & More than 5 years & $3.39 \pm .48$ & \\
\hline \multirow{2}{*}{ Department } & Department with contact with patients & $3.50 \pm .68$ & \multirow{2}{*}{$\begin{array}{l}2.076 \\
(.039)\end{array}$} \\
\hline & Department with no contact with patients & $3.33 \pm .49$ & \\
\hline \multirow{2}{*}{$\begin{array}{l}\text { Respiratory system infection management } \\
\text { education within the last } 1 \text { year }\end{array}$} & Yes & $3.44 \pm .48$ & \multirow{2}{*}{$\begin{array}{l}1.083 \\
(.282)\end{array}$} \\
\hline & No & $3.32 \pm .78$ & \\
\hline \multirow{2}{*}{$\begin{array}{l}\text { Contact with patient suspected of } \\
\text { COVID-19 }\end{array}$} & Yes & $3.40 \pm .44$ & \multirow{2}{*}{$\begin{array}{c}.044 \\
(.965)\end{array}$} \\
\hline & No & $3.40 \pm .63$ & \\
\hline \multirow{2}{*}{ Contact with confirmed COVID-19 patient } & Yes & $3.63 \pm .33$ & \multirow{2}{*}{$\begin{array}{c}.981 \\
(.328)\end{array}$} \\
\hline & No & $3.39 \pm .59$ & \\
\hline
\end{tabular}


Correlations Between COVID-19 Infection Management Knowledge, Importance Level, Extent of Educational Demand, and Performance: The correlations between COVID-19 infection management knowledge, importance level, extent of educational demand, and performance are given in Table 4. The level of execution of COVID-19 infection management had a positive correlation with knowledge of $(\mathrm{r}=.19, p=.007)$ and extent of educational demand for $(\mathrm{r}=.3, p<.001)$ COVID-19.

Table 4. Correlations between Study Variables

\begin{tabular}{|l|c|c|c|}
\hline \multirow{2}{*}{} & Knowledge & Importance level & Extent of educational demand \\
\cline { 2 - 4 } & $\mathbf{r}(\boldsymbol{p})$ & $\mathbf{r}(\boldsymbol{p})$ & $\mathbf{r}(\boldsymbol{p})$ \\
\hline Performance of infection management & .19 & .12 & .33 \\
$(.007)$ & $(.099)$ & $(<.001)$ \\
\hline
\end{tabular}

Factors Affecting Execution of COVID-19 Infection Management: In order to analyze the factors that affect performance of COVID-19 infection management by administrative staff at general hospitals, multiple regression analysis was executed, with the departments in which subjects work, COVID-19 infection management knowledge, and extent of educational demand as the independent variables. Analyzing the tolerance limit and Variance Inflation
Factor (VIF) confirmed the multicollinearity of the independent variables tolerance limit to be in the range of 0.913 0.981, while the VIF value was in the range of 1.019 1.096, thereby demonstrating that no variables were affected by multicollinearity. Analysis results demonstrated that the factors relating to the performance of COVID-19 infection management included the extent of educational demand $(\beta=.372, p<.001)$, with explanatory power of $16.7 \%$ (Table 5 ).

Table 5. Factors Affecting performance of COVID-19 Infection Management

\begin{tabular}{|c|c|c|c|c|c|}
\hline & B & SE & $\beta$ & $\mathbf{t}$ & $\mathbf{p}$ \\
\hline (Constant) & 1.26 & .36 & & 3.51 & .001 \\
\hline Knowledge & .02 & .01 & .11 & 1.53 & .13 \\
\hline Extent of educational demand & .52 & .09 & .37 & 5.29 & $<.001$ \\
\hline Department (Dummy-department with no contact with the patient) & -.09 & .07 & -.09 & -1.39 & .17 \\
\hline
\end{tabular}

\section{Discussion}

This study aimed to provide basic data for the development of infection management educational programs to stop the spread of COVID-19 by assessing the factors that affect the performance of COVID-19 infection management, with administrative staff at general hospitals as the subjects. $66.7 \%$ of subjects received respiratory infection management education during the previous year at the time of the outbreak of the COVID-19 pandemic, with $28.1 \%$ coming in contact with patients suspected of COVID-19 and 3.1\% with confirmed COVID-19 cases. Although it is difficult to make comparisons due to the lack of preceding studies, it can be seen that not only are medical staff coming in contact with suspected or confirmed COVID-19 patients, but also administrative staff. Therefore, it is necessary to provide infection management education for administrative staff at the time of outbreak of new infectious diseases such as COVID-19.

The average score for COVID-19 infection management knowledge of general hospital administrative staff was $14.54 \pm 2.69$ out of a possible 20 points. Although the respective study subjects and infectious diseases differed, the results of surveys of nurses' knowledge of SARS and $\operatorname{MERS}^{(6)}$ were $13.46 \pm 2.97$ out of a possible 20 points, which is similar to the results of this study. However, in a study of nursing college students ${ }^{(10)}$, the average score for the extent of the knowledge of personal protective devices to be used against acute respiratory infectious diseases was 17.83 
out of a possible 20 points, which is higher than the results of this study. Accordingly, it is though that the level of knowledge of administrative staff is similar to that of nurses, since COVID-19 infection management education was executed for all staff at the medical institutions, and information regarding COVID-19 ceaselessly conveyed through mass media. The importance placed on COVID-19 infection management had an average score of $4.09 \pm .88$ out of possible 5 points. Although it is difficult to make comparisons due to lack of preceding studies, it is deemed that administrative staff also consider COVID-19 infection management to be important at the time of the current COVID-19 pandemic. The average score for the extent of educational demand for COVID-19 infection management was $3.66 \pm .34$ out of a possible 4 points, with the highest score for the extent of educational demand for the transmission route and transmission prevention of COVID-19, followed by staff safety, and the putting on and taking off of personal protective devices, while the score for the extent of educational demand on lifting of patient isolation was the lowest. In the study of the extent of educational demand for SARS and MERS information among nurses ${ }^{(5)}$, the scores were in the order of treatment and nursing, isolation method, clinical symptoms, and method of putting on personal protective devices, thereby displaying differences to the results of this study. That is, the medical staff displayed a higher extent of educational demand in the domains of treatment and nursing, since patient treatment and nursing are their main roles. On the other hand, since the roles of administrative staff include allocation of hospital rooms and supply of goods for patients rather than treatment, the scores for the extent of educational demand for transmission route and transmission prevention were the highest. In addition, while nurses displayed lower scores for the extent of educational demand on the use of personal protective devices, since they use these frequently, it is thought that administrative staff displayed higher scores for this domain since they use protective devices only during special situations. The average score for the performance of COVID-19 infection management was $3.40 \pm .58$ out of a possible 4 points, with higher scores for the subordinate domains in the order of infection management by staff, respiratory etiquette, and personal protective devices.

There were differences in the scores for the execution of COVID-19 infection management depending on the department that the administrative staff worked in, with the staff working in departments with contact with patients displaying higher scores. It is thought that the frequency of execution of the guidelines for COVID-19 infection management would be higher in the case of more frequent contact with patients. In terms of the correlations among COVID-19 infection management knowledge, importance level, extent of educational demand, and extent of performance, the extent of performance of COVID-19 infection management was found to be higher with higher levels of COVID-19 knowledge and extent of educational demand. According to the results of this study, 28.1\% of the administrative staff came in contact with COVID19-suspected patients. Accordingly, COVID infection management needs to be executed for administrative staff, and it is anticipated that the spread of infectious diseases within medical institutions can be stopped more quickly and easily if the level of the execution of infection management were to increase through more extensive acquisition of accurate COVID-19 infection management knowledge. A factor affecting performance of COVID-19 infection management was the extent of educational demand, with explanatory power of $16.7 \%$. It was found that the level of execution of COVID-19 infection management is higher with a higher extent of educational demand. Therefore, it is important to assess the extent of educational demand of administrative staff regarding infectious diseases other than COVID-19, since there is very high possibility of such future outbreaks.

\section{Conclusion and Acknowledgements}

This study was significant in that it assessed COVID-19 infection management knowledge and the extent of educational demand among general hospital administrative staff, providing basic data for the development of COVID-19 infection management education programs for administrative staff by analyzing the factors that affect the performance of COVID-19 infection management. The extent of educational demand was the factor imparting the most significant effect on the execution of COVID-19 infection management by general hospital administrative staff. The extent of educational demand for the domains of transmission path, transmission prevention, and staff safety against COVID-19 was high. Therefore, it is necessary to include the domains of transmission method, insolation guidelines, and staff infection management of COVID-19 at the time of the development of COVID-19 infection management educational programs in the future. 
This study has several limitations and the recommended directions for future studies to supplement these limitations are suggested below. First, general hospitals in a single region with easier accessibility were selected in consideration of the response range of the questionnaire. Accordingly, there could be limitations in terms of generalizing the results of this study to all general hospital administration staff throughout the country. Second, it is important to review and confirm the subordinate domains of extent of educational demand in order to increase execution of infection management. Accordingly, it would be necessary in future to execute a follow-up study for the verification of effects after developing individual educational programs for each of the subordinate domains.

Source Funding: This paper was supported by the Konyang University Research Fund in the second half of 2020

\section{Conflict of Interest: None}

Ethical Clearance: This research has ethical clearance from the Institutional Review Board of Konyang University (KYU-2020-139-01)

\section{References}

1. Zhu N, Zhang D, Wang W, Li X, Yang B, Song $\mathrm{J}$, et al. A novel coronavirus from patients with pneumonia in China. The New England Journal of Medicine.2019;382:727-733. https://doi. org/10.1056/NEJMoa2001017

2. Choi SH. Preventive measures during outbreak of coronavirus disease 2019. Korean Journal of Medicine,2020;95(3):134-140. https://doi. org/10.3904/kjm.2020.95.3.134

3. Cowling BJ, Park M, Fang VA, Wu P, Leung GM, $\mathrm{Wu}$ JT. Preliminary epidemiology assessment of MERS-CoV outbreak in South Korea, May to June 2015. Euro Surveillance, 2015;20(25). https://doi. org/10.2807/1560-7917.es2015.20.25.21163
4. June KJ, Choi ES. Infection control of hospital nurses: cases of middle east respiratory syndrome. Korean Journal of Occupational Health Nursing. 2016;25(1):1-8. https://doi.org/10.5807/ kjohn.2016.25.1.1

5. Choi YE, Lee ES. A Study on knowledge, attitude, infection management intention \& educational needs of new respiratory infectious disease among nurses who unexperienced NRID (SARS \& MERS). Journal of the Korean Academia-Industrial Cooperation Society, 2019;20(2):721-731. https:// doi.org/10.5762/KAIS.2019.20.2.721

6. Kim OS. Knowledge and compliance with prevention of respiratory tract infection among workers in geriatric facilities. Korean Journal of Nosocomial Infection Control. 2015; 20(2):61-69.

7. Rajakaruna SJ, Liu W, Ding Y, Cao G. Strategy and technology to prevent hospital-acquired infections: Lessons from SARS, Ebola, and MERS in Asia and West Africa. Military Medical Research.2017;4:32-36. https://doi.org/10.1186/ s40779-017-0142-5

8. Danzmann L, Gastmeier P, Schwab F, Vonberg RP. Health care workers causing large nosocomial outbreaks: a systematic review. BMC Infectious Disease. 2013;13:98-108. https://doi. org/10.1186/1471-2334-13-98

9. Parmeggiani C, Abbate R, Marinelli P, Angelillo IF. Healthcare workers and health care-associated infections: knowledge, attitudes, and behavior in emergency departments in Italy. BMC Infectious Diseases.2010;10:35-44. https://doi. org/10.1186/1471-2334-10-35

10. Kim BH, Kang HY. Knowledge, perception, and attitude related to personal protective equipment of student nurses: acute respiratory infections. Journal of the Korea Academia-Industrial Cooperation Society.2019;20(12):139-147. https://doi. 